\title{
LABOUR MARKET AND PECULIARITIES OF INFORMAL EMPLOYMENT IN THE CONTEXT OF THE GLOBAL PANDEMIC
}

\author{
MURMAN TSARTSIDZE \\ Doctor of Economic Sciences, Associate Professor \\ Ivane Javakhishvili Tbilisi State University, \\ Academician of the Georgian Academy of Economic Sciences, Georgia \\ murman.tsartsidze@tsu.ge
}

\section{NATELA LATSABIDZE}

Academic Doctor, invite professor

Ivane Javakhishvili Tbilisi State University, Georgia

natela.latsabidze@tsu.ge

\begin{abstract}
In terms of overcoming the socio-economic problems in Georgia, great importance is attached to efficient functioning of the labour market. It must ensure a radical change the sphere of social-labour relations, accordingly, efficient overcoming the chronic unemployment in the country and efficient employment of labour force. The results of the research confirms, that adequate labour market of normal market environment is still on the formation stage with serious problems and challenges which is developed in Georgia. In 1990s at the initial stage of formation the national labour market difficult economic situation which was in the country, give a push negative developments in the field of labour relations, among which before nowadays invincible challenge is the growing trend of informal employment. It can be said, that this one took on a large-scale character in the current period of financial and economic crisis caused by the global pandemic. In fact, the informal sector in Georgia has become an independent segment of the labour market, which has a significant impact on employment of the population, standard of living and on the overall process of economic development. Informal employment, as an important study socio-economic event and phenomenon, assess its level and scale, it can be said convincingly that it will not lose its relevance for many years. Negative consequences of informal employment, needs analysis and evaluation factors affecting the influx of economically active population into the informal sector of the labour market. Also its important discuss and evaluate the positive aspects of informal employment in the short term, which manifested in facilitation reasons socio-economic and psychological consequences of unemployment.

In the work researched certain aspects of informal employment, analyzed its forms, faces, identified peculiarities of informal employment and rated its scale against the background of the global pandemic. Substantiated, that informal segment of the labour market requires depth research and evaluation. By the research identified the main factors for the growth and development of employment in the informal sector of economy in the Georgian labour market, from which we must distinguish: high levels and scale of poverty in the country, chronic unemployment and inefficient employment; insecurity social principle of justice in the field of employment; equal employment opportunities for job seekers; lack of proper conditions for equal realization of labour rights; population low availability on different types of labour market services and events, inadequate rates of economic development; reduced demand for labour, accordingly limited employment opportunities and prospects; low level and quality of development of vocational education system, which not fully focused on labour market demands, social protection of the unemployed, none of insurance mechanism; difficulties in training and retraining the unemployed; low level of remuneration; low level of stimulation, motivation and labour organization, underdevelopment of labour market infrastructure and its management information system, problems in the field of management and regulation of social-labour relations, which connected to the development of the legislative-normative base, lack of a complete mechanism for controlling the working conditions of employees and etc. The main results of the research are summarized in the concluding part of the work.
\end{abstract}

KEYWORDS: LABOUR MARKET, INFORMAL SEGMENT OF LABOUR MARKET, UNEMPLOYMENT, INFORMAL EMPLOYMENT, PANDEMIC.

For citation: Tsartsidze, M., Latsabidze, N., (2021). Labour Market and Peculiarities of Informal Employment in the Context of the Global Pandemic. Globalization and Business. 12, 123-132. (In Georgian). https://doi.org/10.35945/gb.2021.12.017 


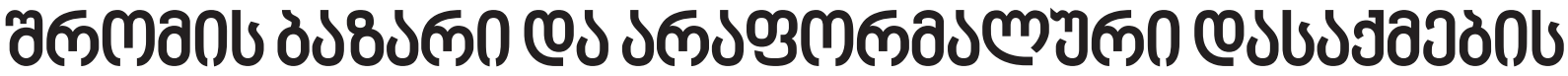

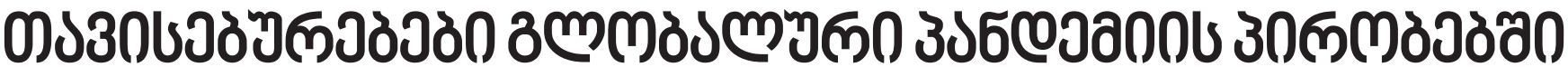

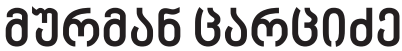

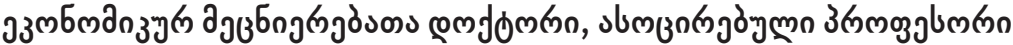

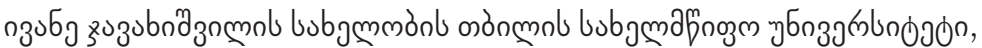

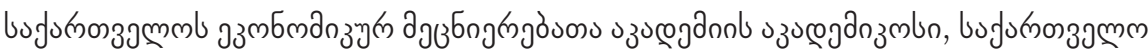 \\ murman.tsartsidze@tsu.ge
}

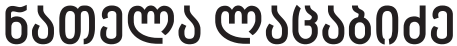

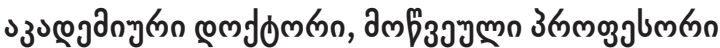

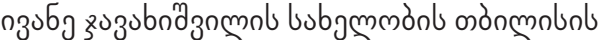

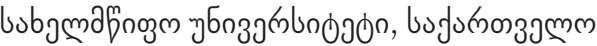

natela.latsabidze@tsu.ge

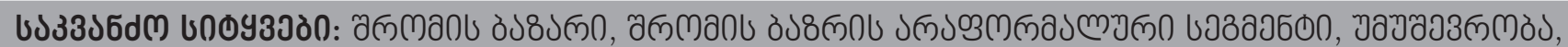

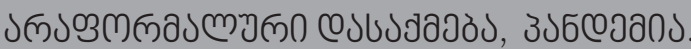

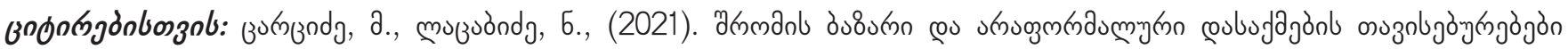

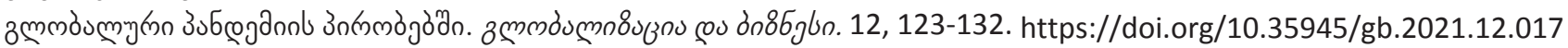

\section{əงเ১З১ल0}

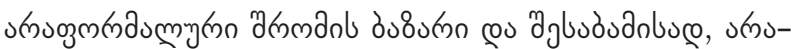

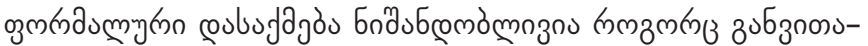

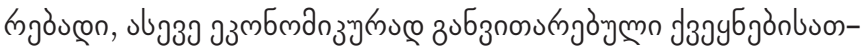

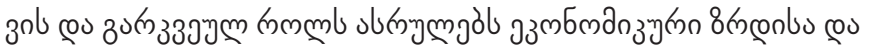

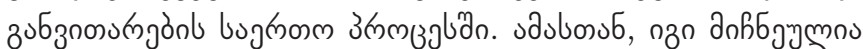

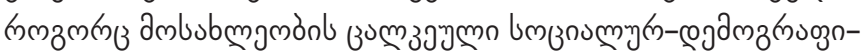

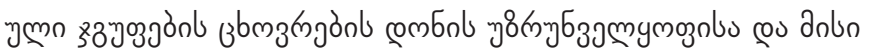

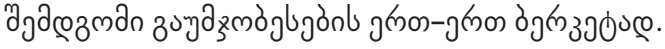

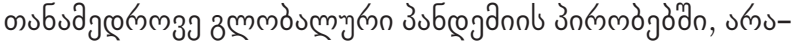

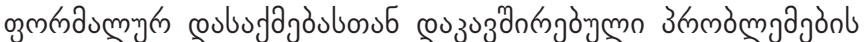

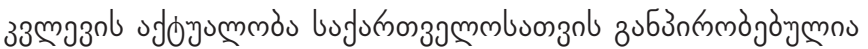

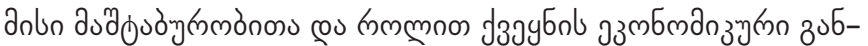

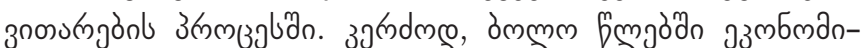

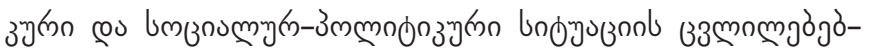
du (Abesadze, 2020; Bedianashvili, 2018; Bedianashvili \& Maglakelidze, 2021; Gaganidze, 2018; Gelashvili et al., 2012;

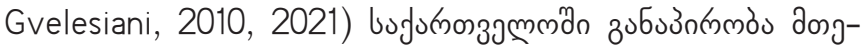

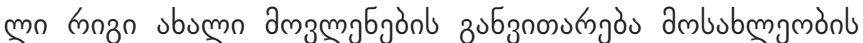

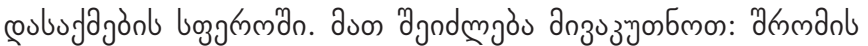

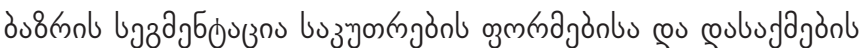

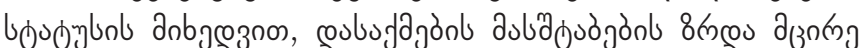

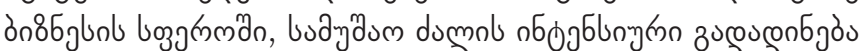

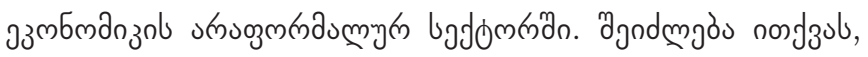

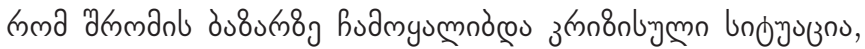

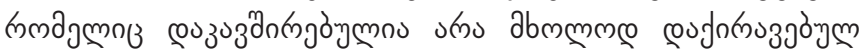

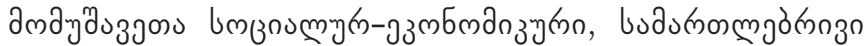

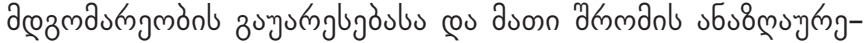

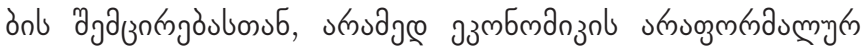

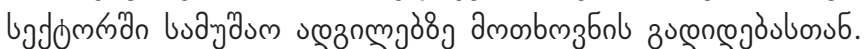

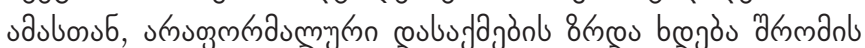

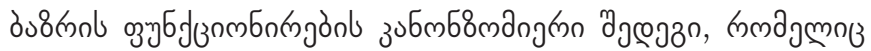

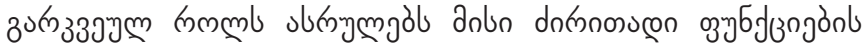
फुग

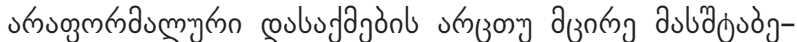

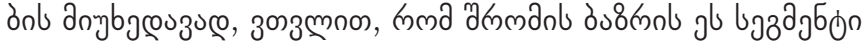

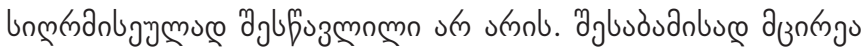

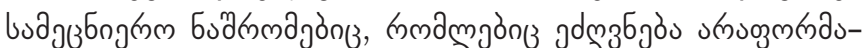

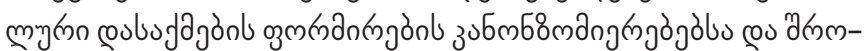

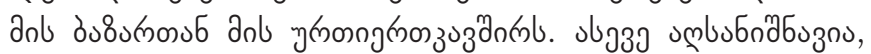

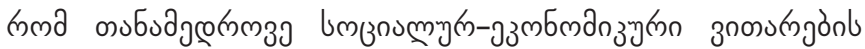

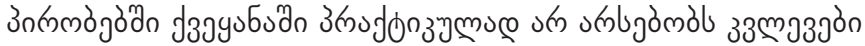

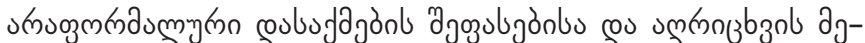

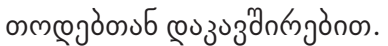

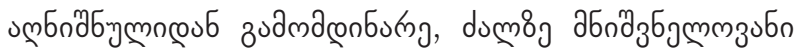

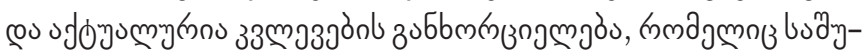

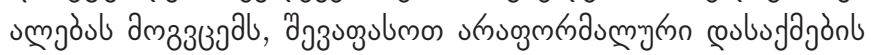




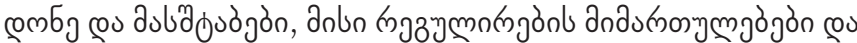

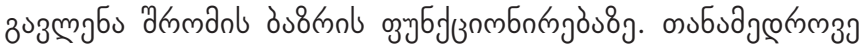

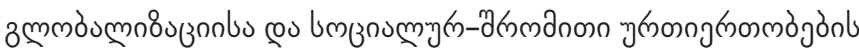

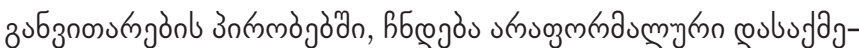
on ๒ øुm

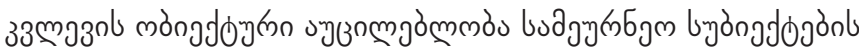

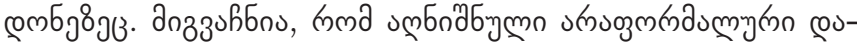

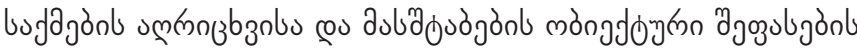

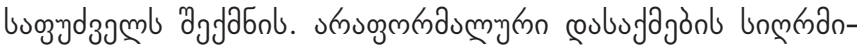

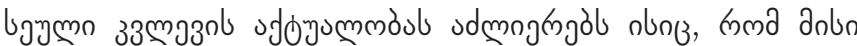

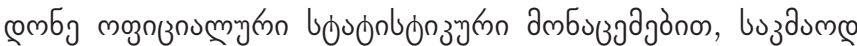
dugummo - budyumme J3ayubudn 35.0\% 2020 famb (http://

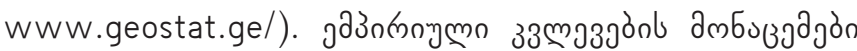

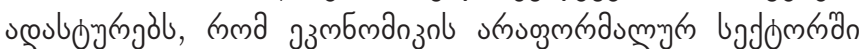

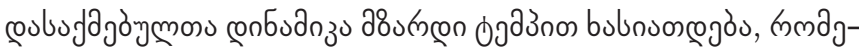

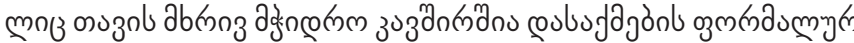

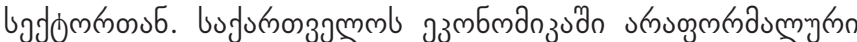

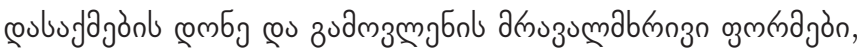

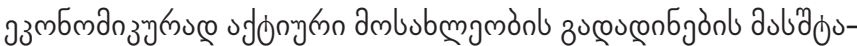

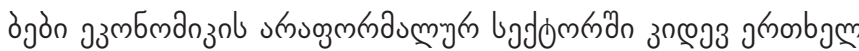

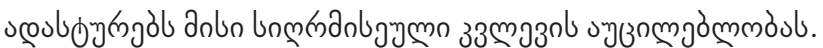

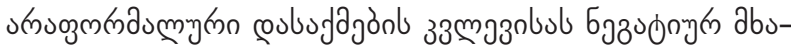

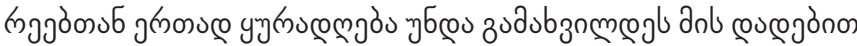

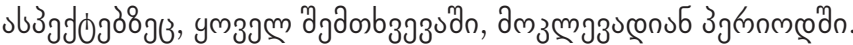

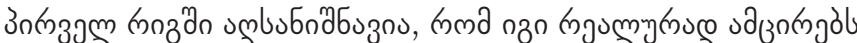

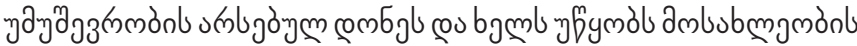

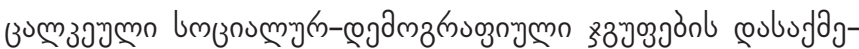

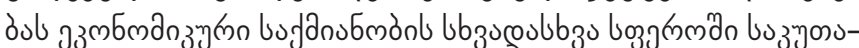

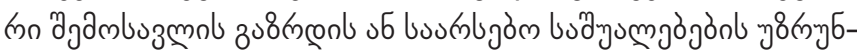

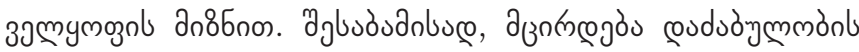

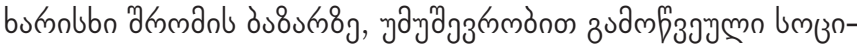

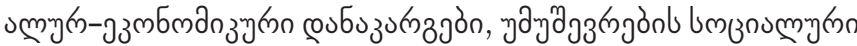

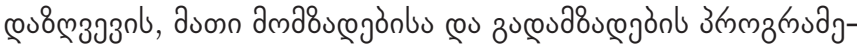

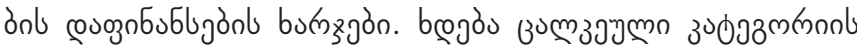

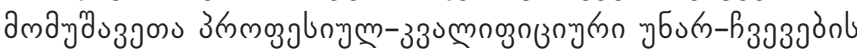

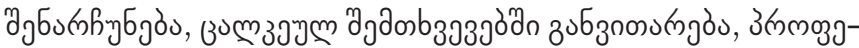

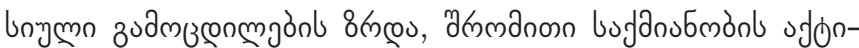

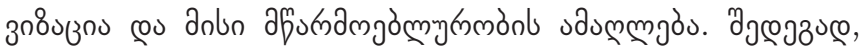

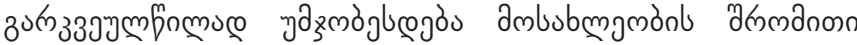

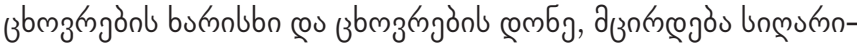

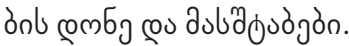

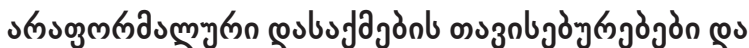

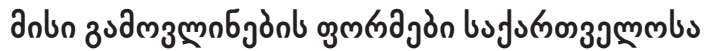 एo bu8}

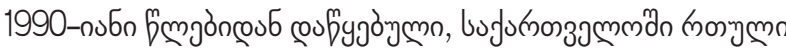

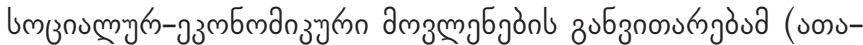

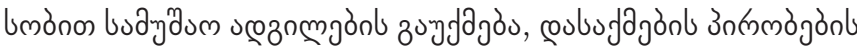

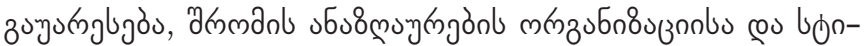

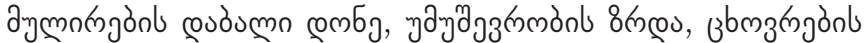

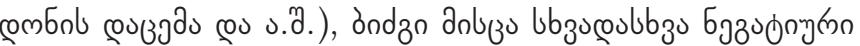

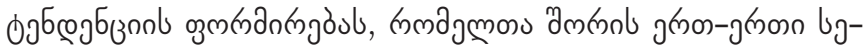

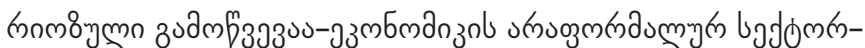

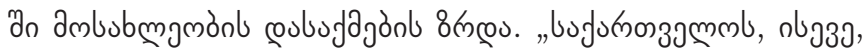

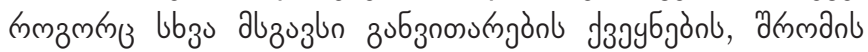

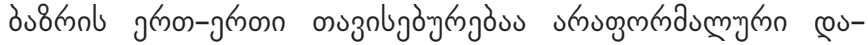

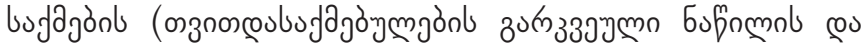

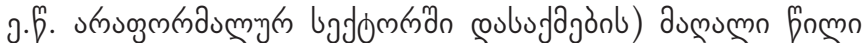

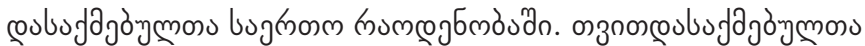

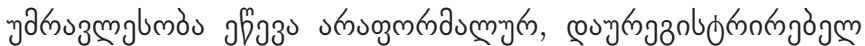

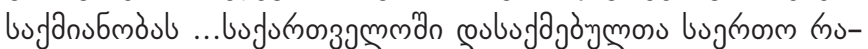

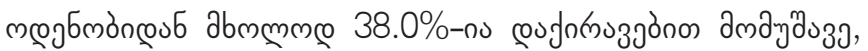

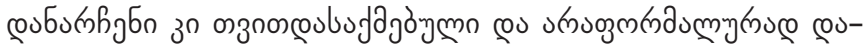

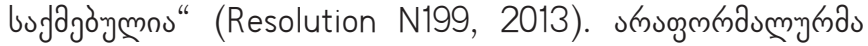

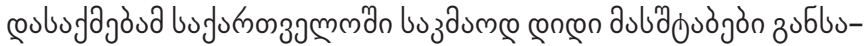

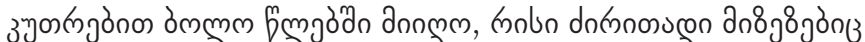

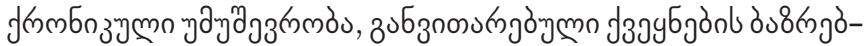

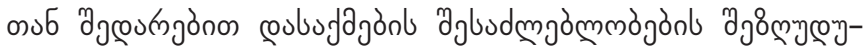

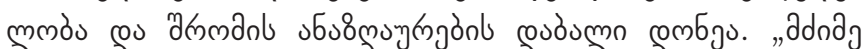

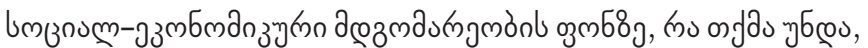

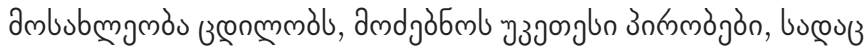

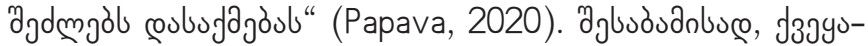

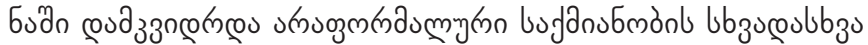

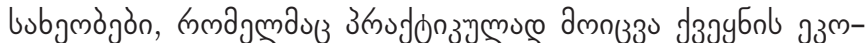

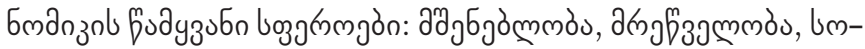

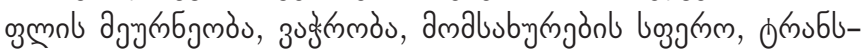

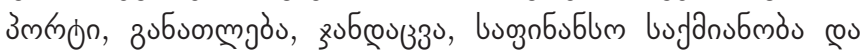

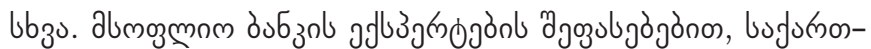

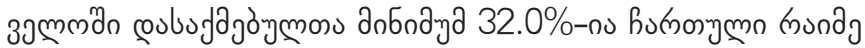

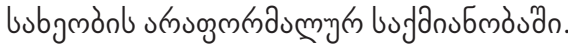

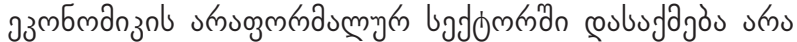

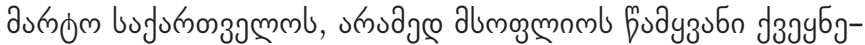

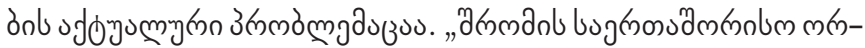

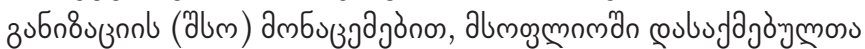

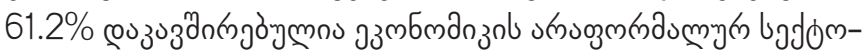

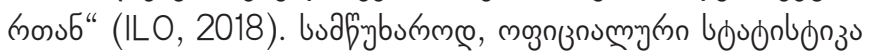

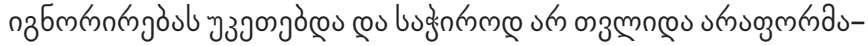

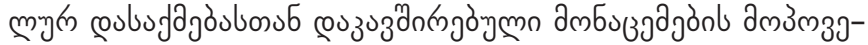

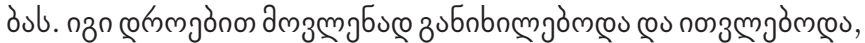

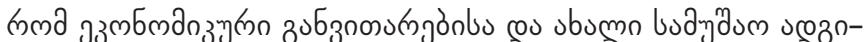

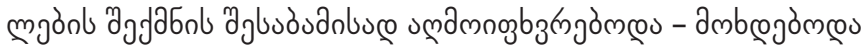

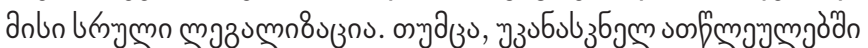

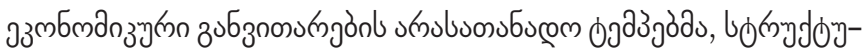

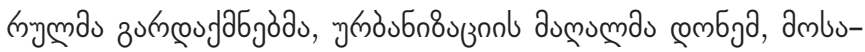

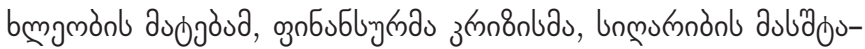

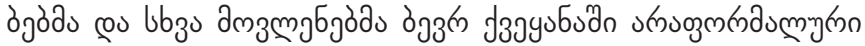

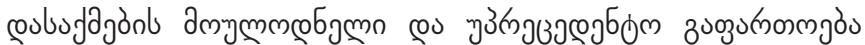

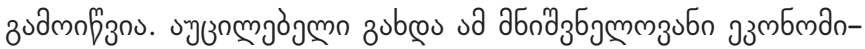

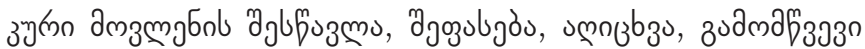




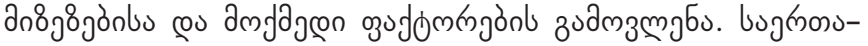

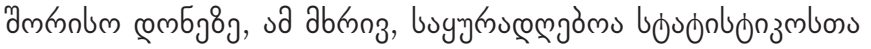

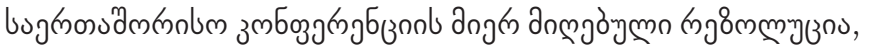

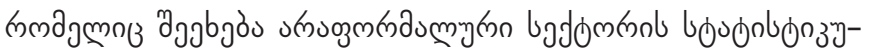
mn zublus

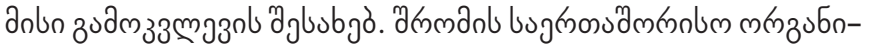

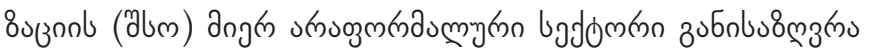

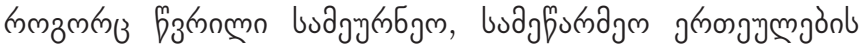

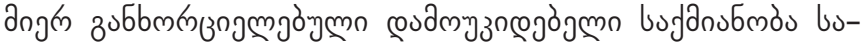

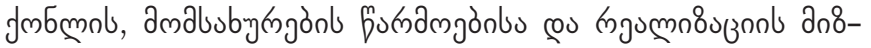

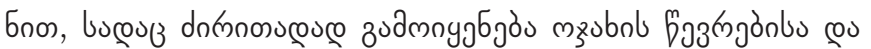

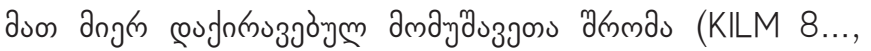

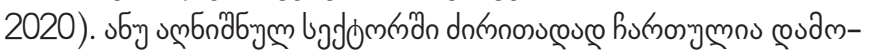

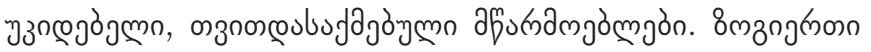

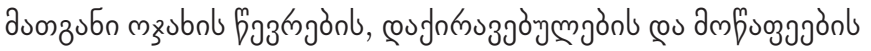

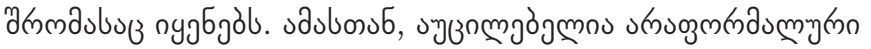

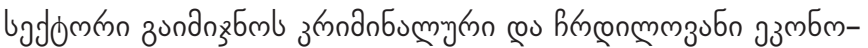

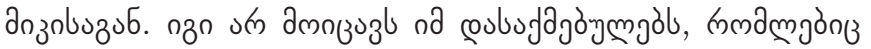

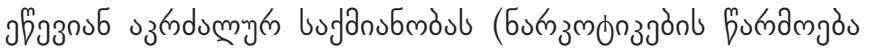

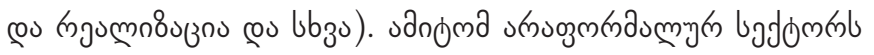

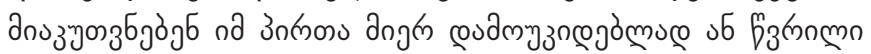

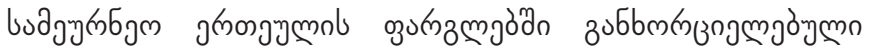

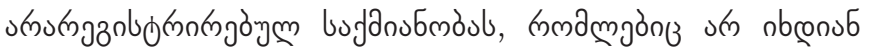

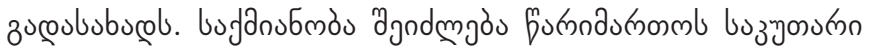

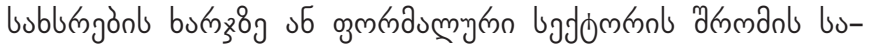

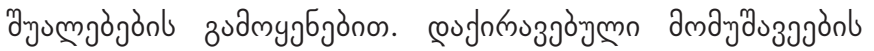

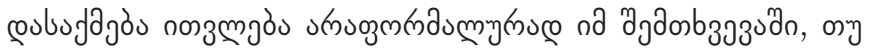

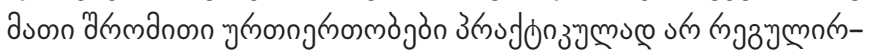

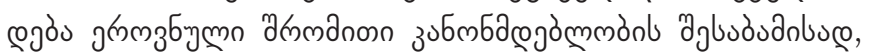

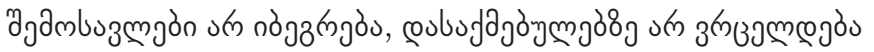

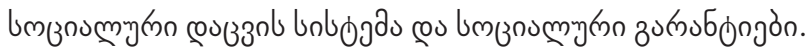

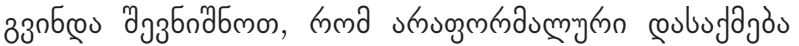

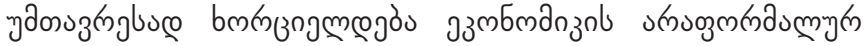

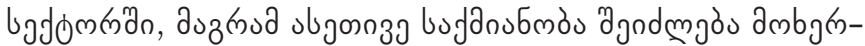

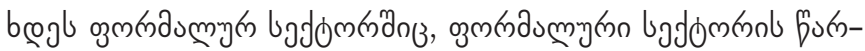

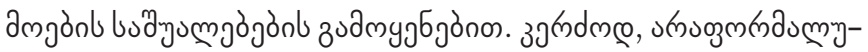

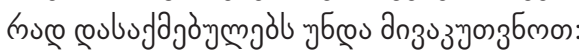

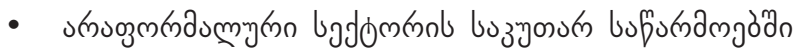

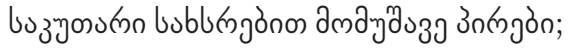

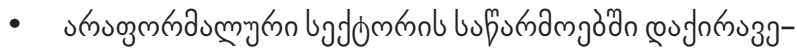

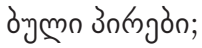

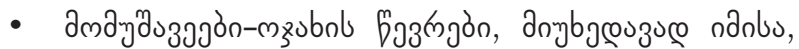

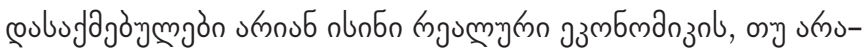

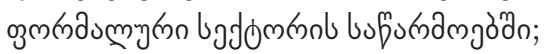

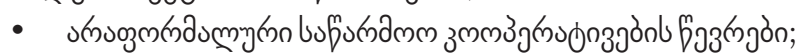

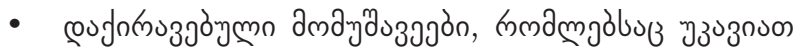

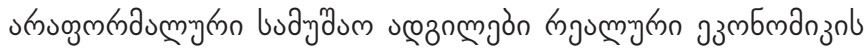
usfurmamgìn;

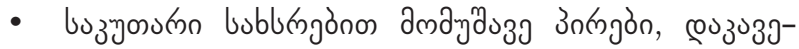

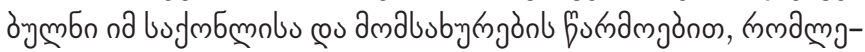

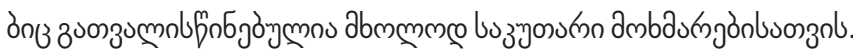

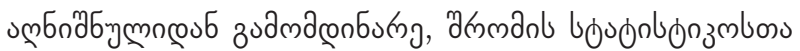

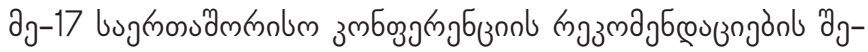

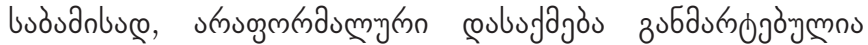

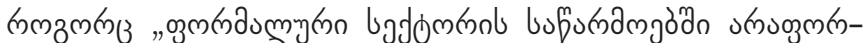

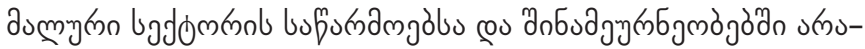

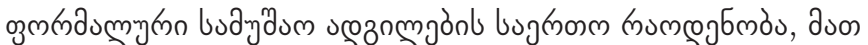

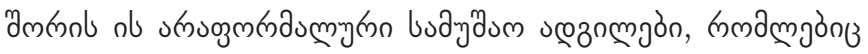

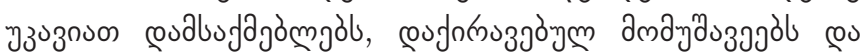

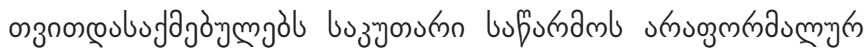

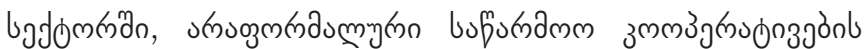

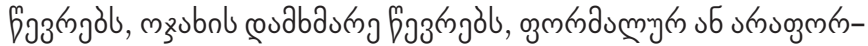

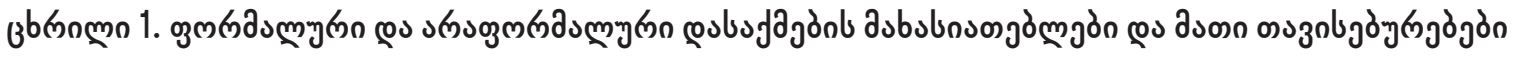

\begin{tabular}{|c|c|c|}
\hline 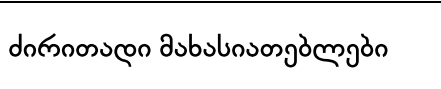 & 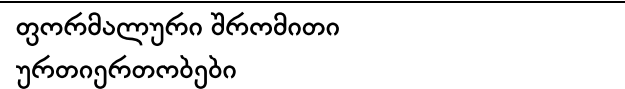 & 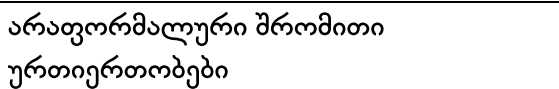 \\
\hline 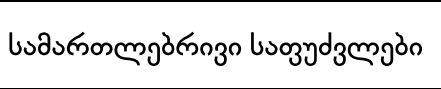 & 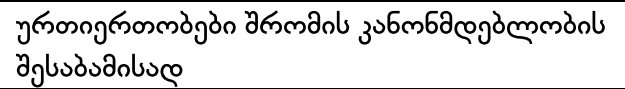 & 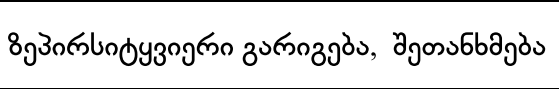 \\
\hline e elusfajònl an8s6n & 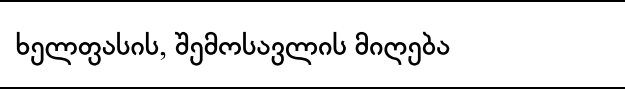 & 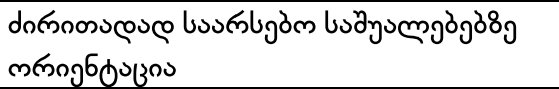 \\
\hline 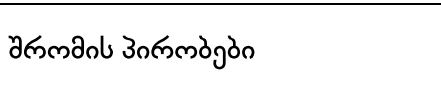 & 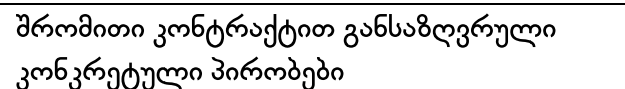 & 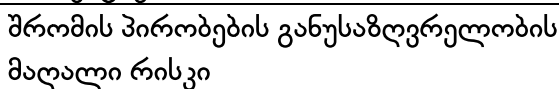 \\
\hline ymmanl cosk30 & 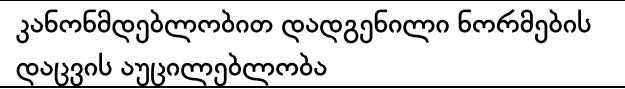 & 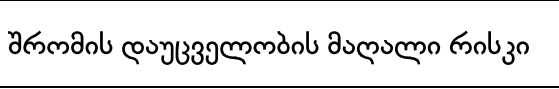 \\
\hline 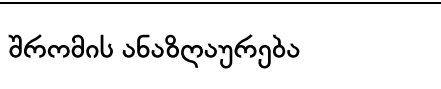 & 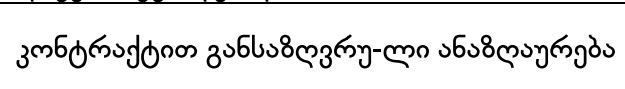 & 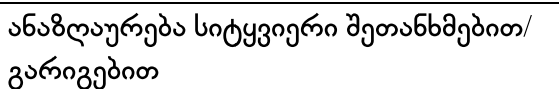 \\
\hline 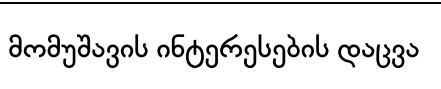 & 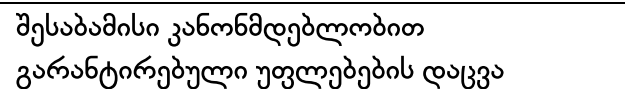 & 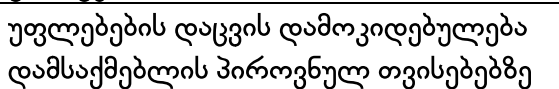 \\
\hline 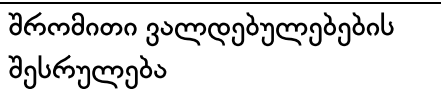 & 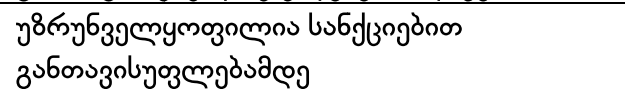 & 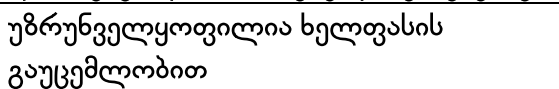 \\
\hline
\end{tabular}

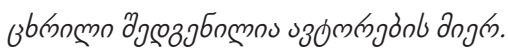




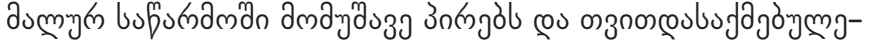

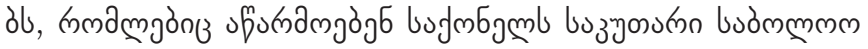

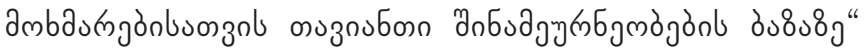
(KILM 8..., 2020).

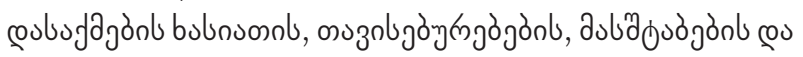

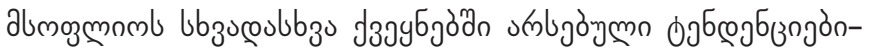

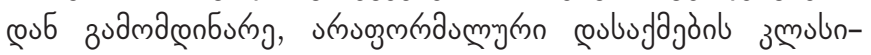

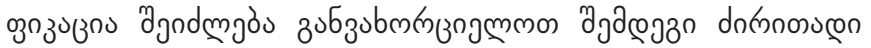

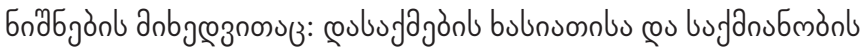

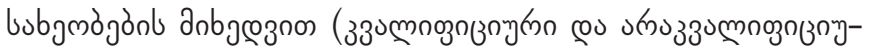

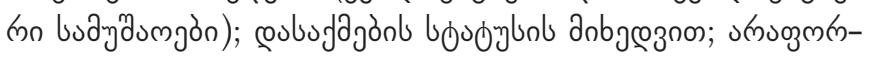

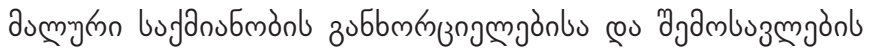

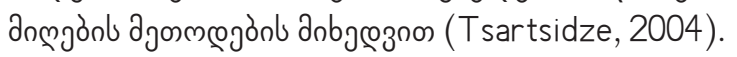

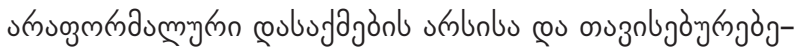

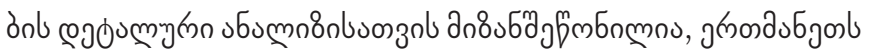

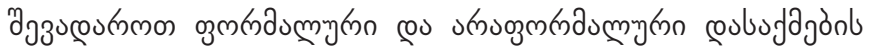

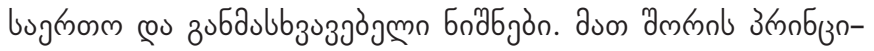

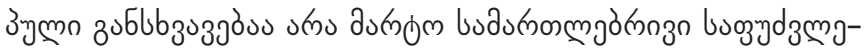

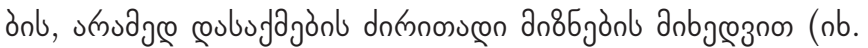

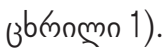

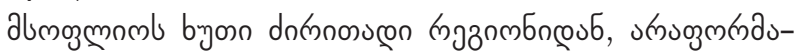

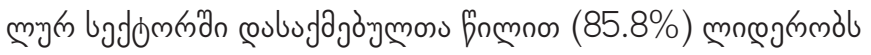

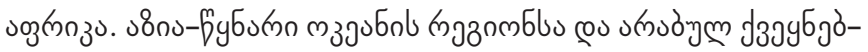
In uल

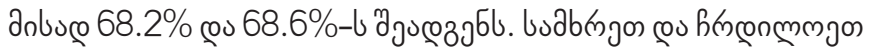

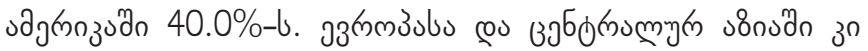

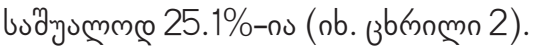

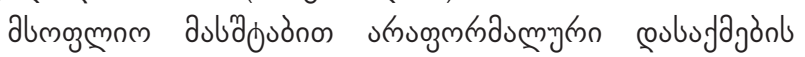

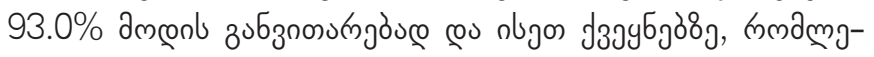

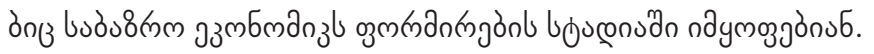

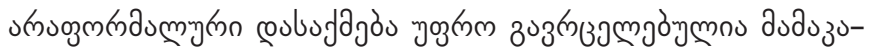

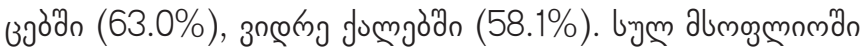

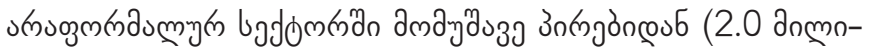

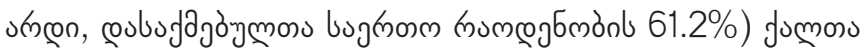

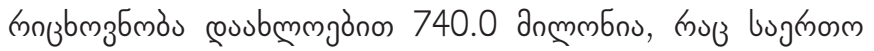

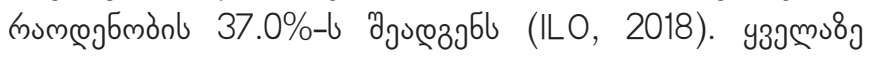

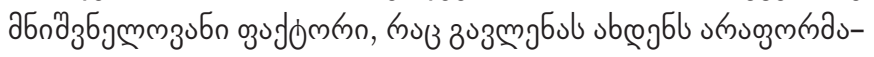

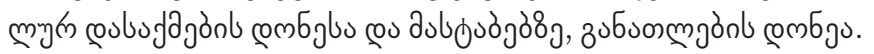

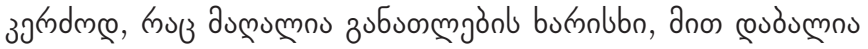

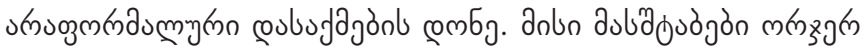

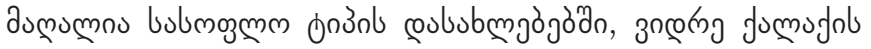

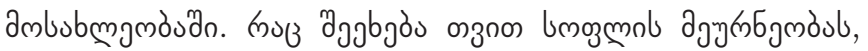

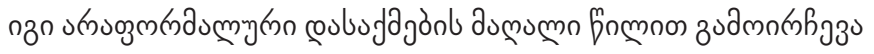

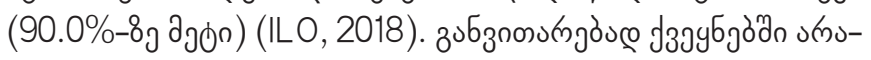

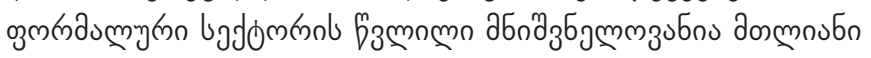

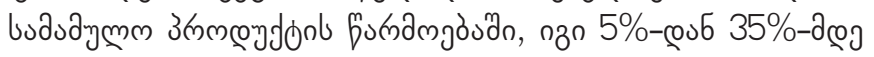

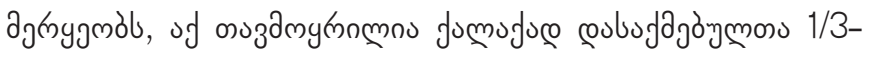

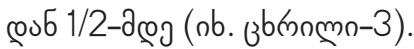

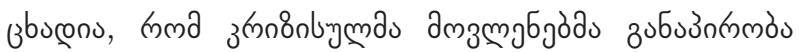

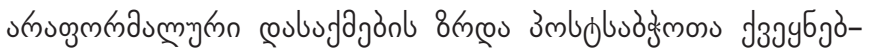

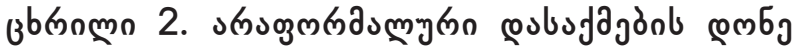

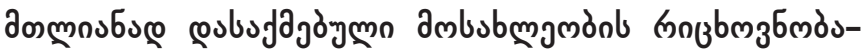

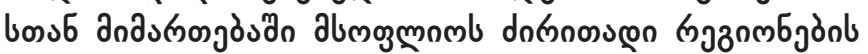

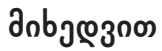

\begin{tabular}{|c|c|c|}
\hline $\mathrm{N}$ & 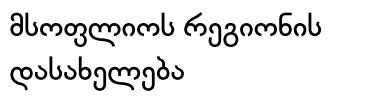 & 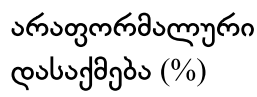 \\
\hline 1 & 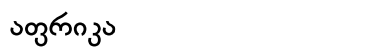 & $85.8 \%$ \\
\hline 2 & 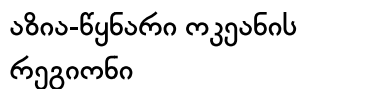 & $68.2 \%$ \\
\hline 3 & 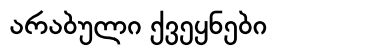 & $68.6 \%$ \\
\hline 4 & 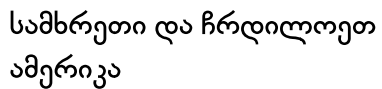 & $40.0 \%$ \\
\hline 5 & 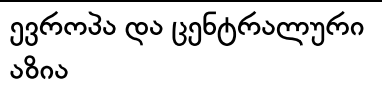 & $25.1 \%$ \\
\hline & 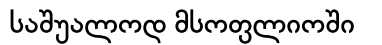 & $61.2 \%$ \\
\hline
\end{tabular}

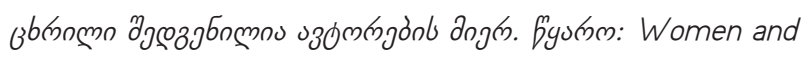
men in the informal economy (2018): A statistical picture. Third edition. Report. International Labour Office, Geneva. Copyright OInternational Labour Organization. First published 2018 Web PDF: 9789221315810[ISBN]. https://www.ilo.org/global/ publications/books/WCMS_626831/lang--en/index.htm.

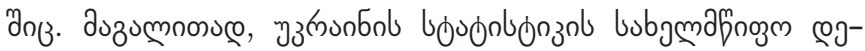

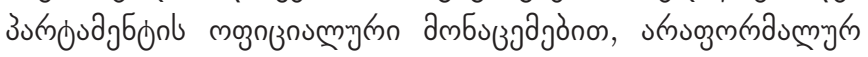
bədom

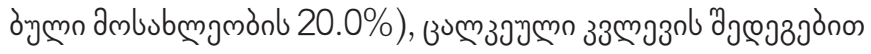

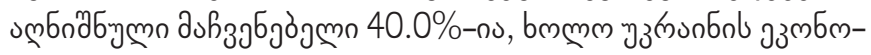

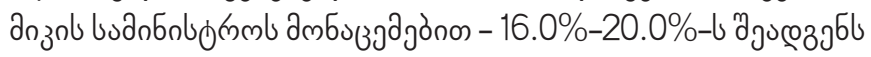
(Informal, 2020).

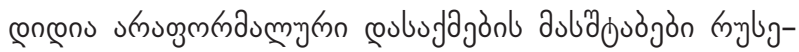

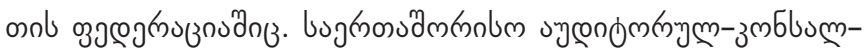
onб

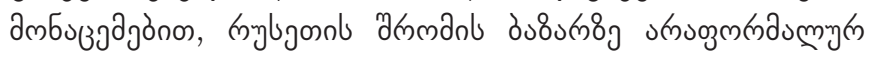

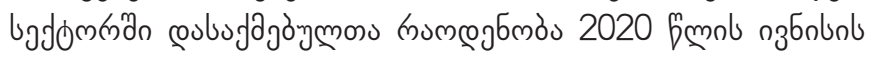

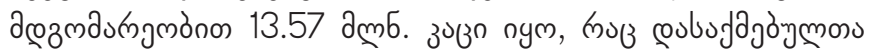

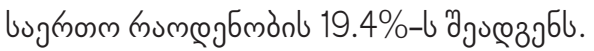

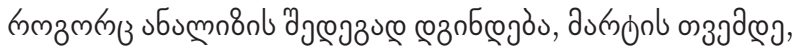

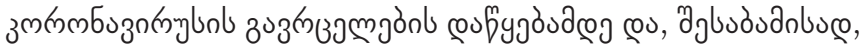

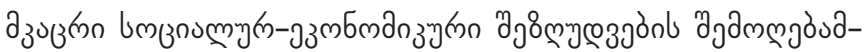

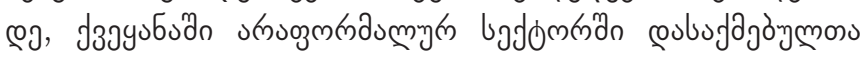

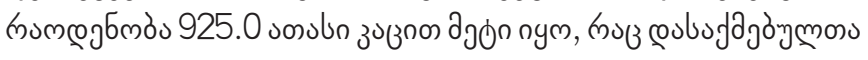

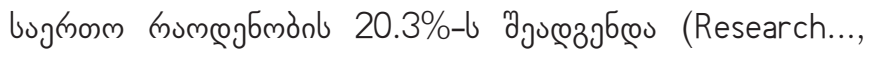
2020).

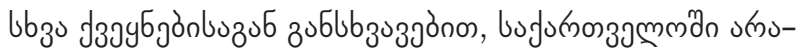

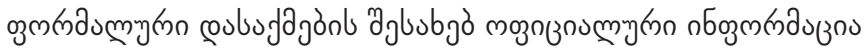




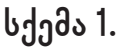

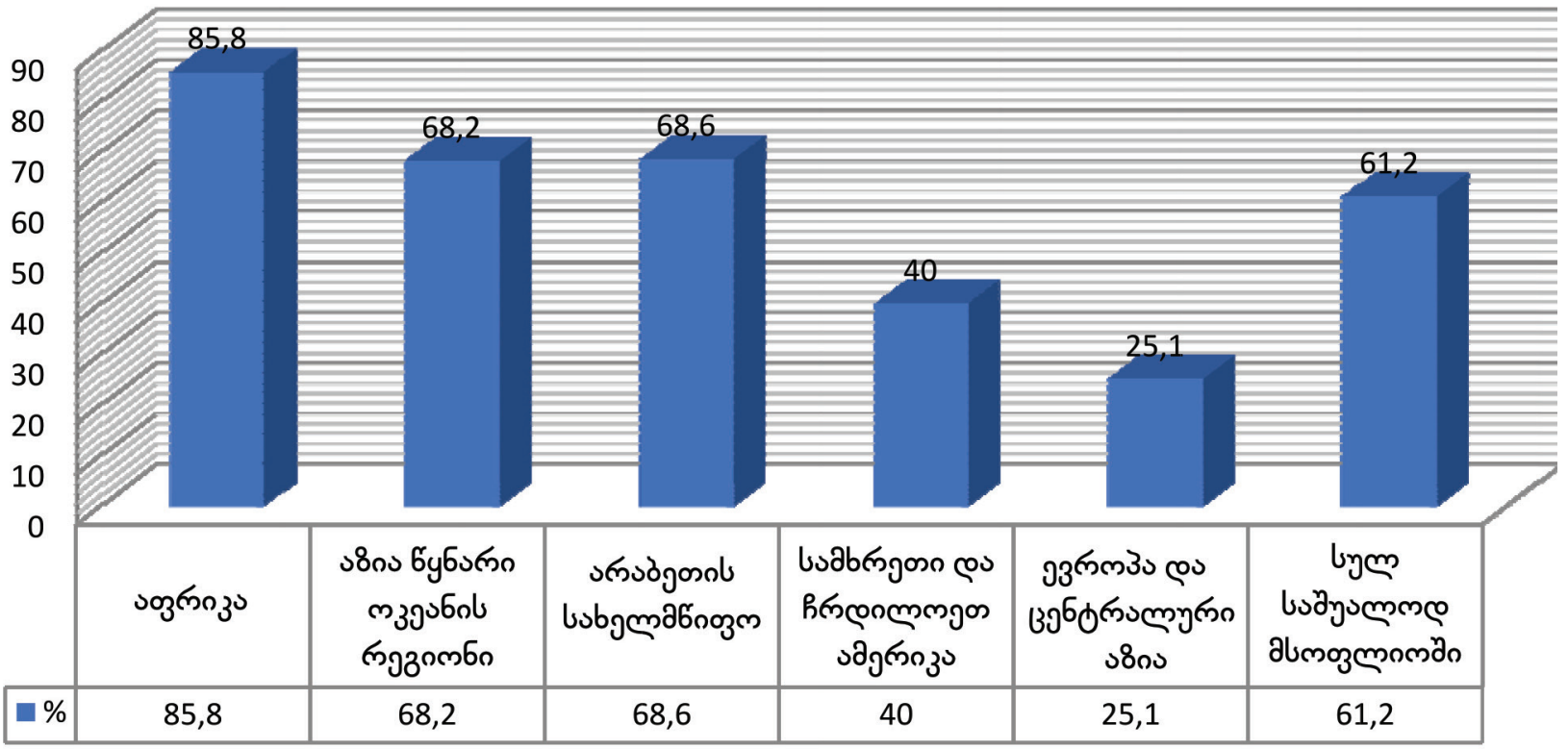

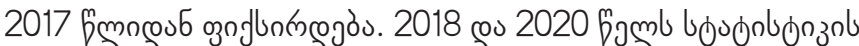

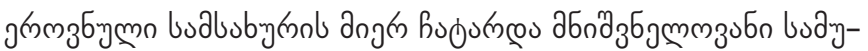

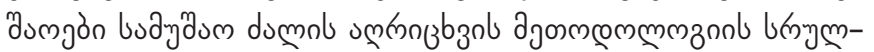

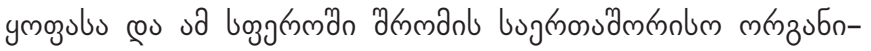

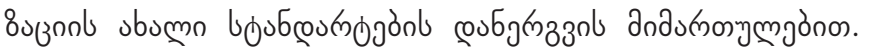

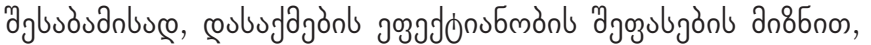

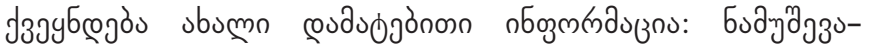

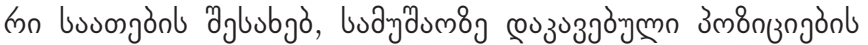

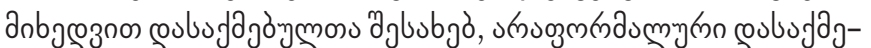
onb gybubgod (nb. zbrinmon 4).

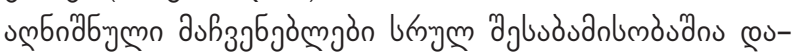

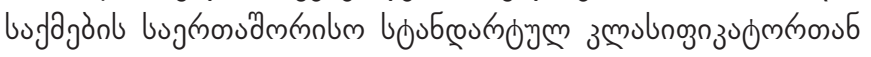

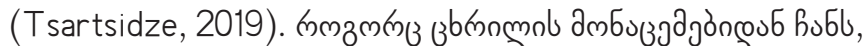

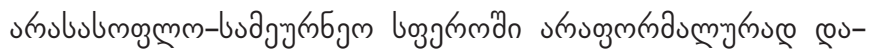

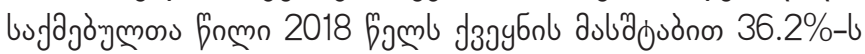

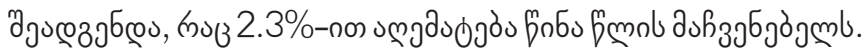

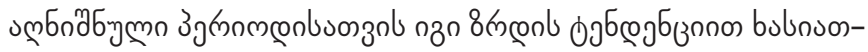

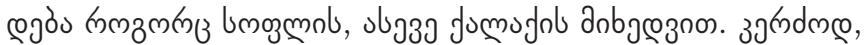

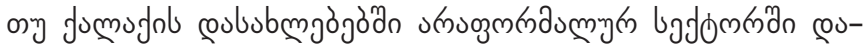

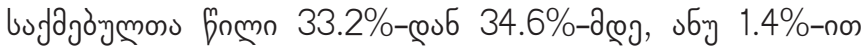

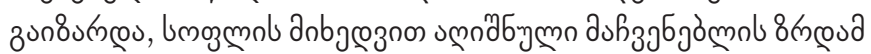

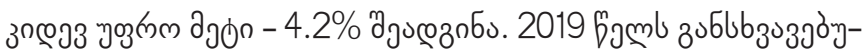

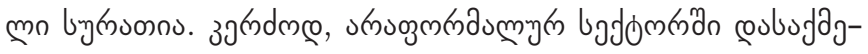

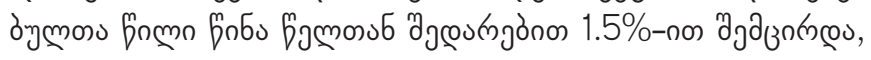

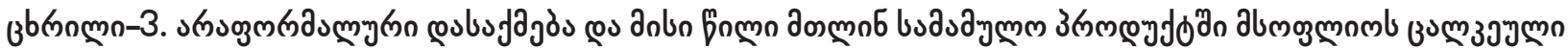

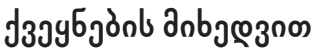

\begin{tabular}{|c|c|c|c|}
\hline$d_{330 y \Delta 60}$ & 6пmо GDP-дю (\%) & j30yง6s & 6оmо GDP-Әо (\%) \\
\hline 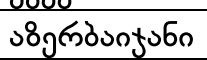 & 59,3 & ग310 momos & $\overrightarrow{14,0}$ \\
\hline dymmfrylo & 19,1 & 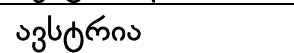 & 9,0 \\
\hline jlombjos & 18,5 & domanos & 22,5 \\
\hline yu8sbgoso & 34,2 & cos6os & 18,3 \\
\hline mod3 300 & 34,8 & önб goso & 18,9 \\
\hline mogdग3 & 25,2 & buog mutaggoso & 14,9 \\
\hline дmmеmзs & 37,7 & 39ตวงธกง & 14,9 \\
\hline frylgoso & 41,0 & 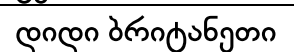 & 14,0 \\
\hline Dु mon6s & 47,3 & ludghrd6goso & 29,0 \\
\hline 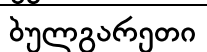 & 32,7 & 'dumos & 27,3 \\
\hline 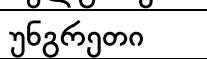 & 28,4 & os3mbos & 11,1 \\
\hline Змलmøболо & 13,9 & $y_{30300}$ & 19,9 \\
\hline 6yanбyos & 18,3 & 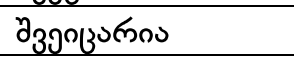 & 8,1 \\
\hline lemm3 39000 & 10,2 & دə⿱一𫝀口 & 8,9 \\
\hline
\end{tabular}

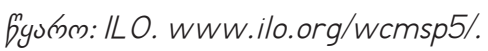




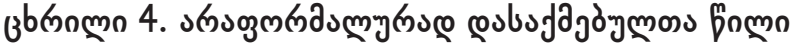

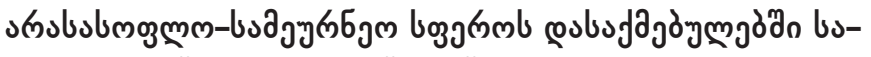

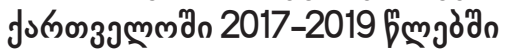

\begin{tabular}{|c|c|c|c|}
\hline \multirow[t]{2}{*}{ 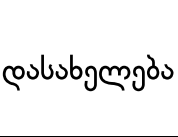 } & \multicolumn{3}{|c|}{ 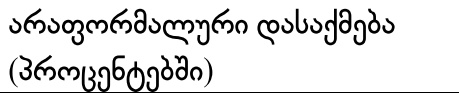 } \\
\hline & 2017 & 2018 & 2019 \\
\hline jumo & $29.2 \%$ & $29.8 \%$ & $29.2 \%$ \\
\hline дง & $37.9 \%$ & $41.5 \%$ & $39.3 \%$ \\
\hline jumodo & $33.2 \%$ & $34.6 \%$ & $33.8 \%$ \\
\hline lмозэмо & $35.8 \%$ & $40.0 \%$ & $36.8 \%$ \\
\hline byem & $33.9 \%$ & $36.2 \%$ & $34.7 \%$ \\
\hline
\end{tabular}

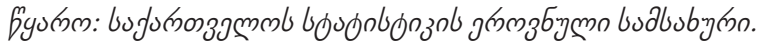

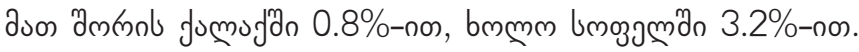

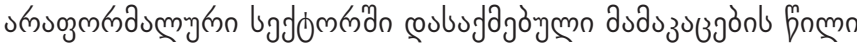

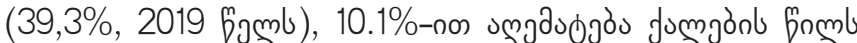

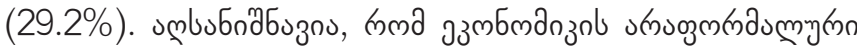

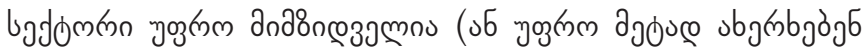

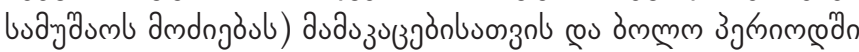

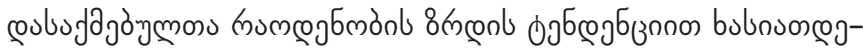

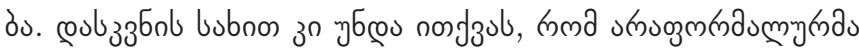

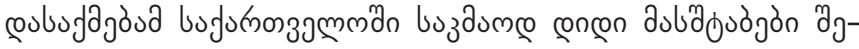

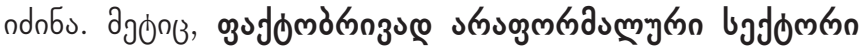

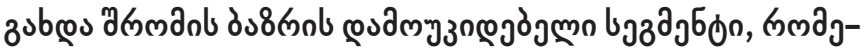
мпз

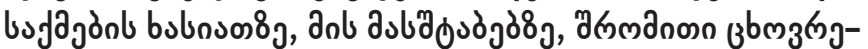

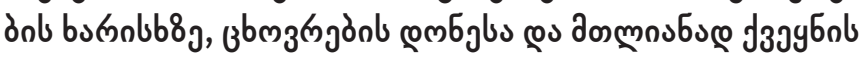

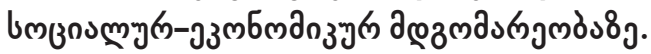

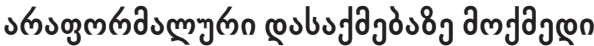

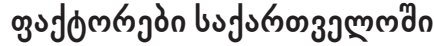

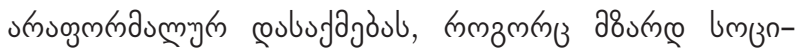

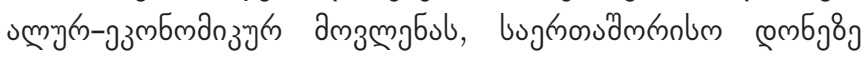

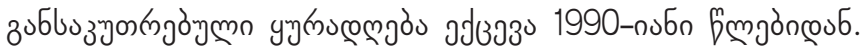

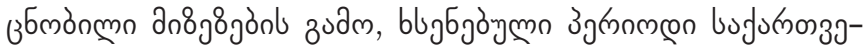

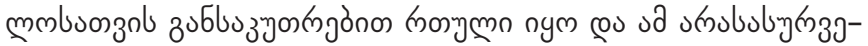

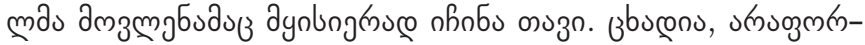

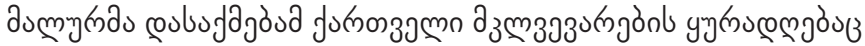

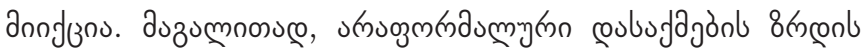

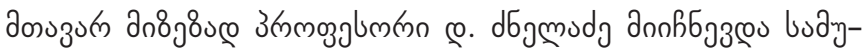

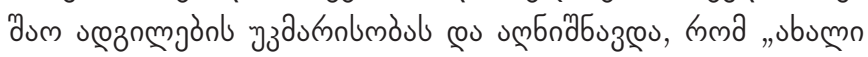

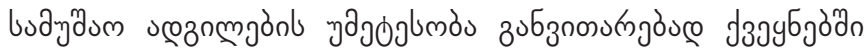

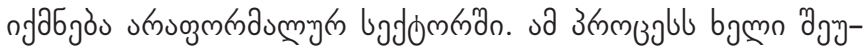

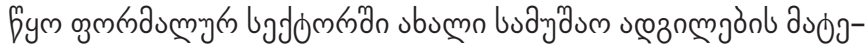

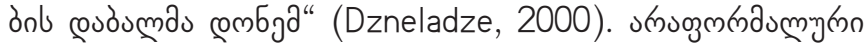

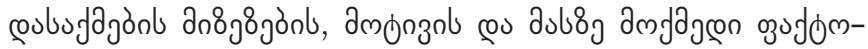

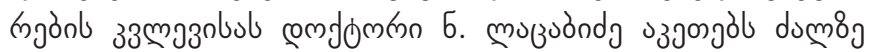

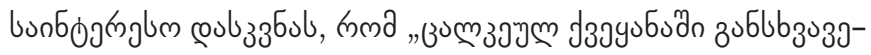

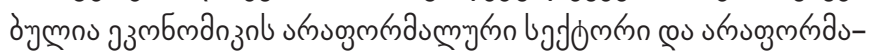

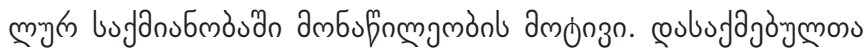

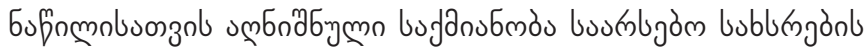

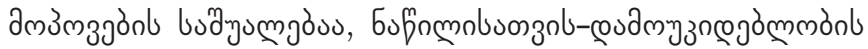

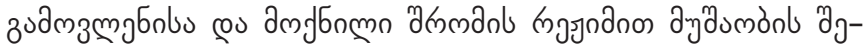

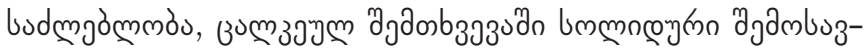

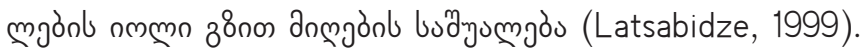

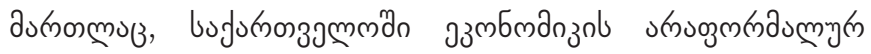

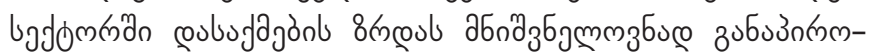

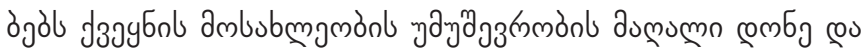

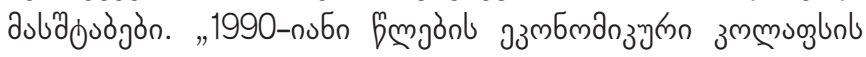

เงวว১ 2.

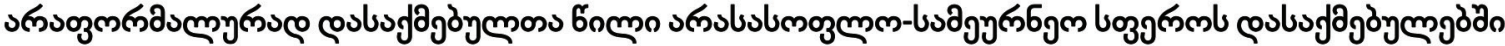

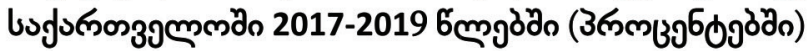

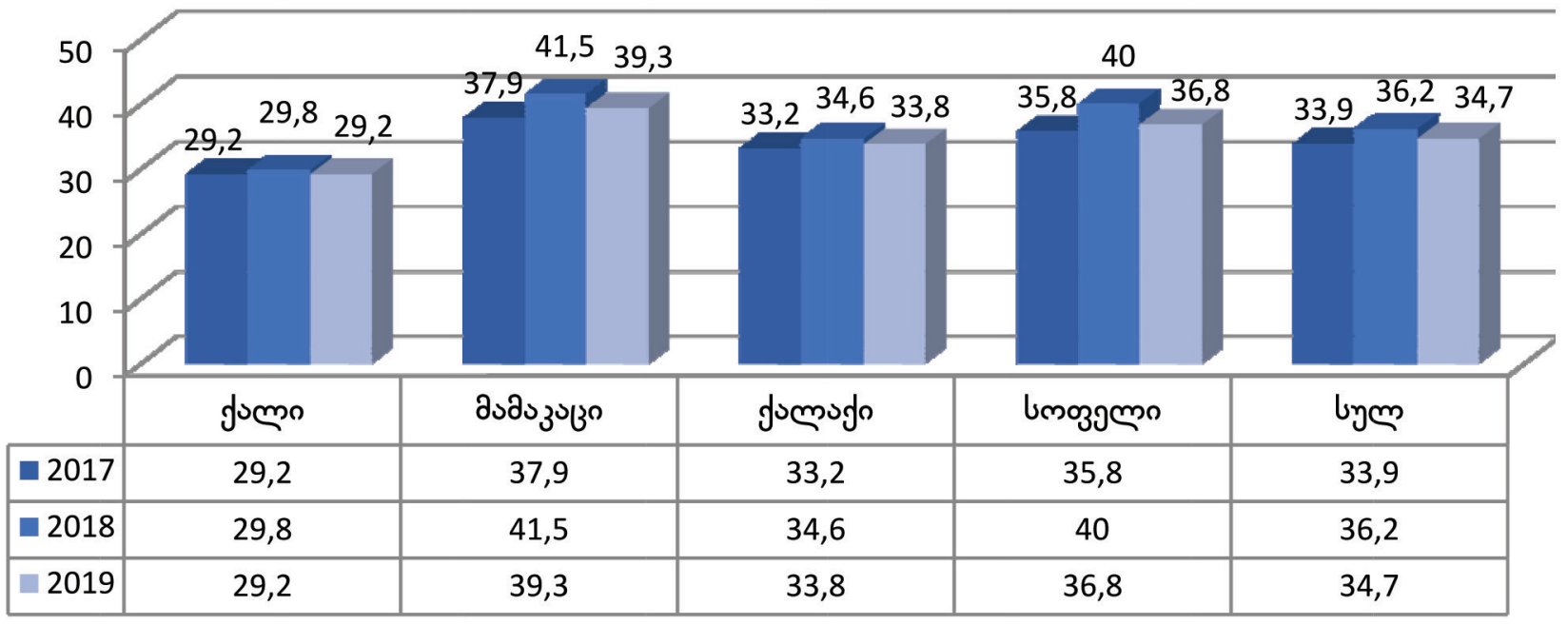




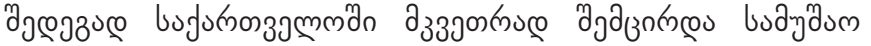

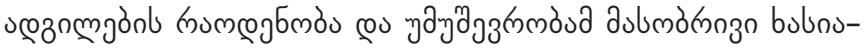

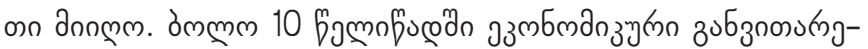
onl dn dnnosucon as

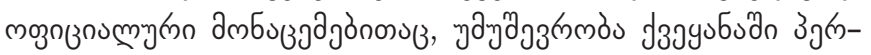

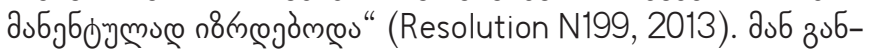

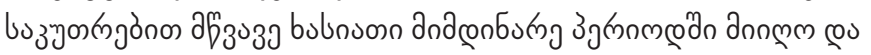

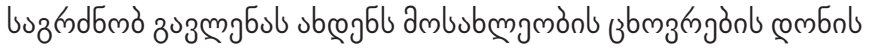

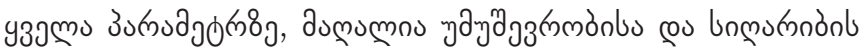

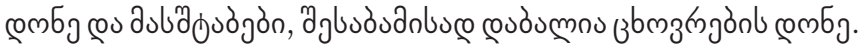

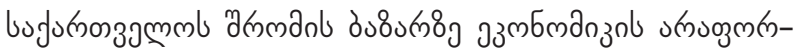

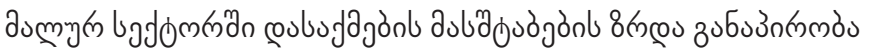

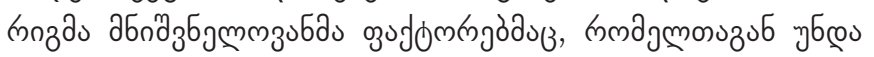

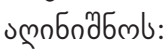

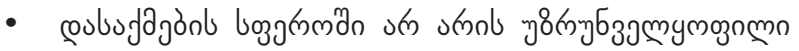

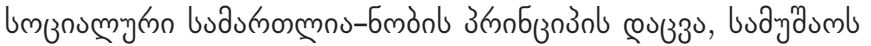

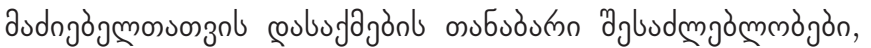

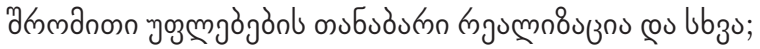

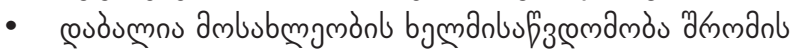

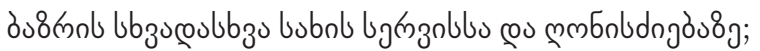

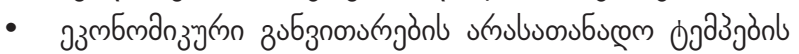

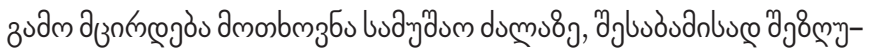

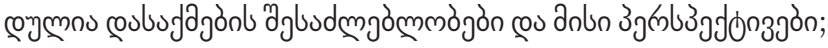

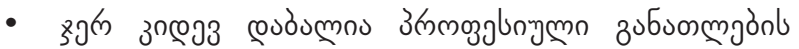

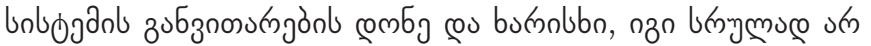

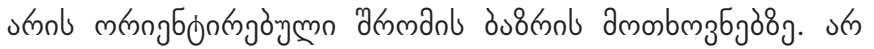

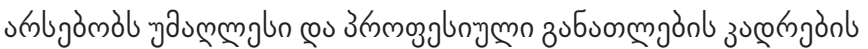

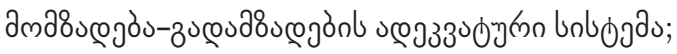

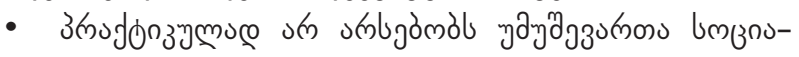

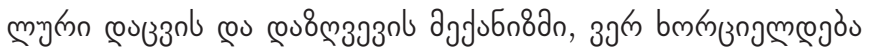
avon zu@ buogyd $39 \mathrm{sm} 8 \mathrm{j} ;$

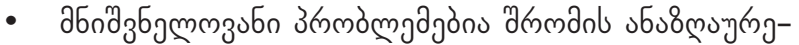

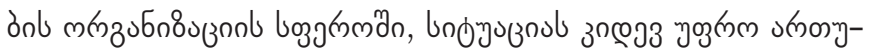

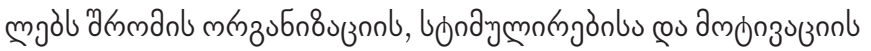
coudumn combg;

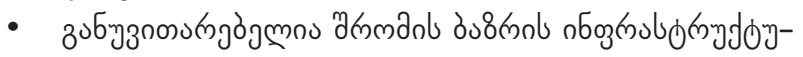

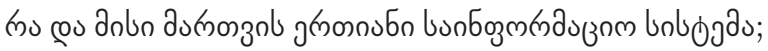

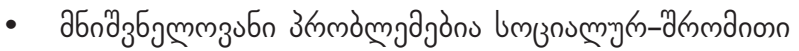

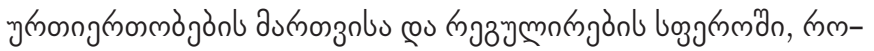

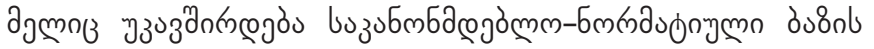

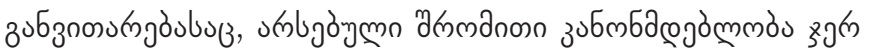

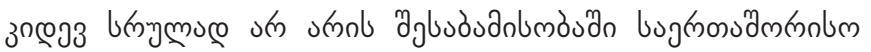
boubcounojojosos:

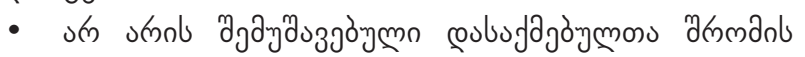

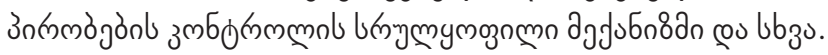

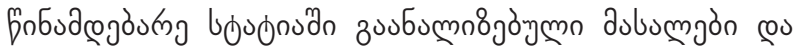

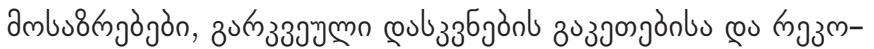

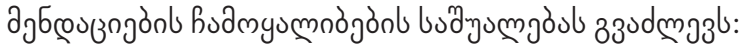

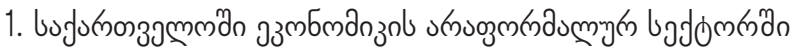

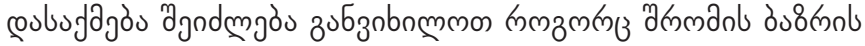

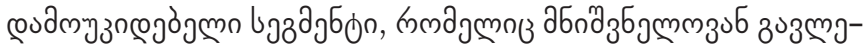

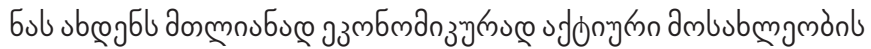

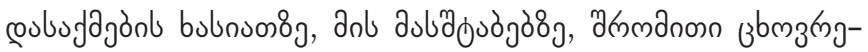

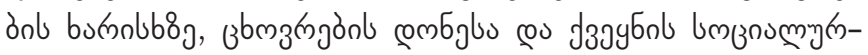

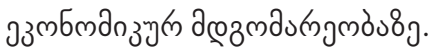

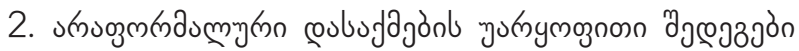

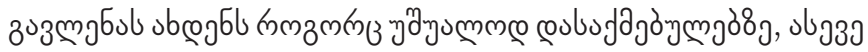

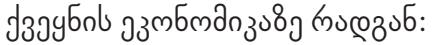

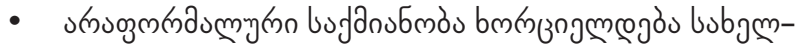

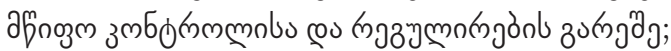

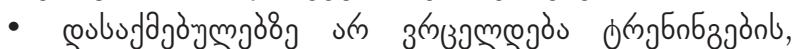

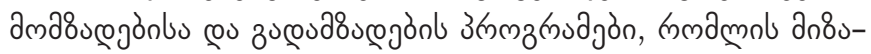

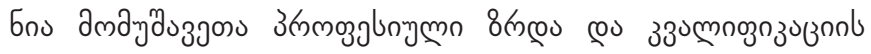

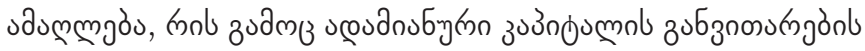

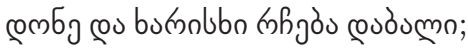

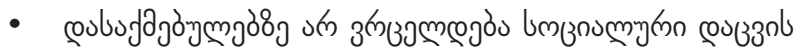

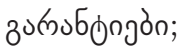

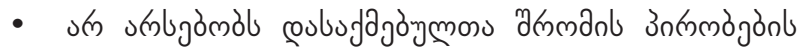

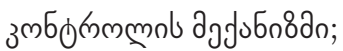

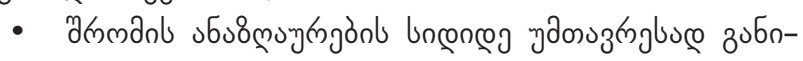

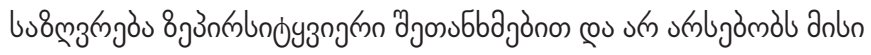

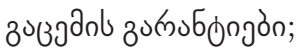

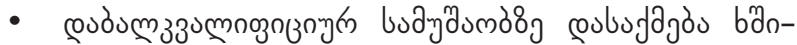

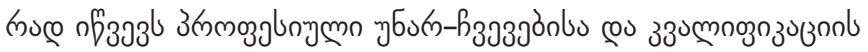
cosdzgnongoub;

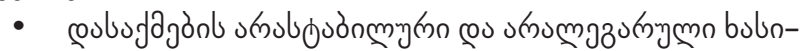

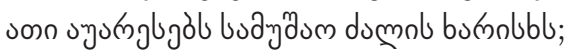

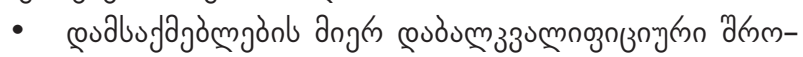

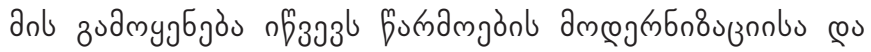

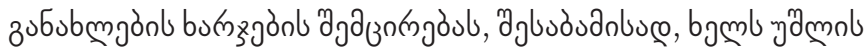

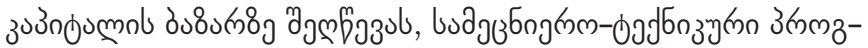

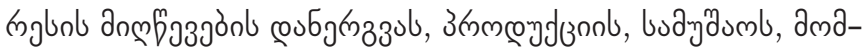

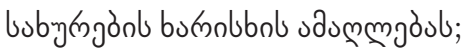

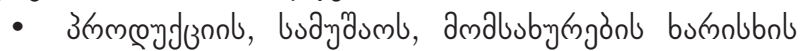

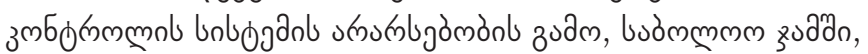

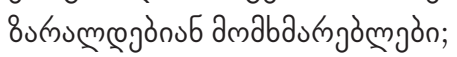

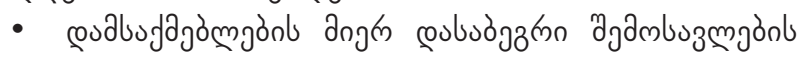

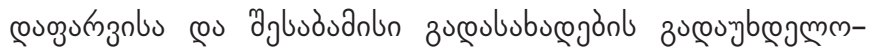

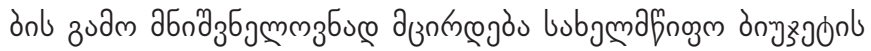
zдаmbuzmgòn.

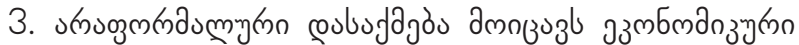

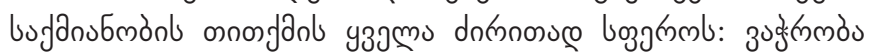

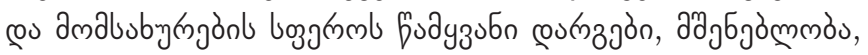

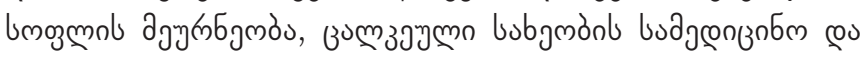
buzubaubsonm

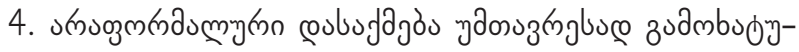

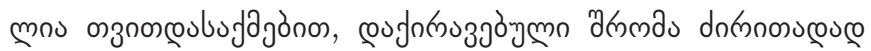

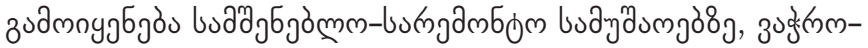

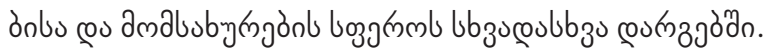




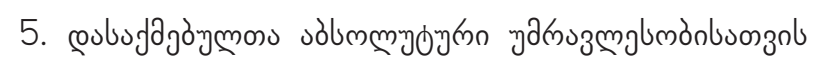

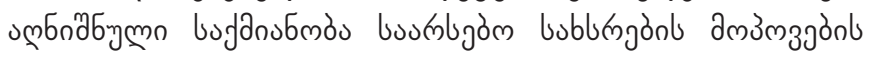

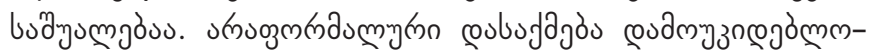

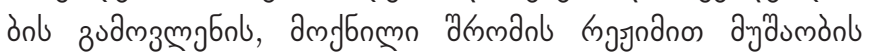

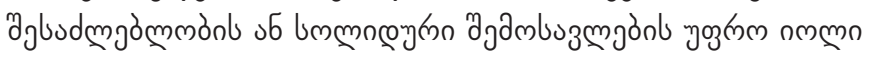

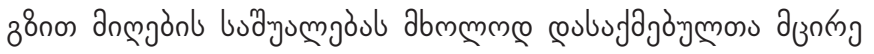

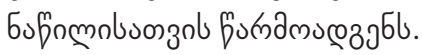

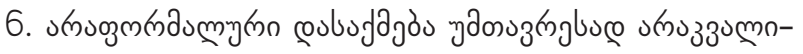

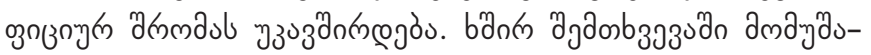

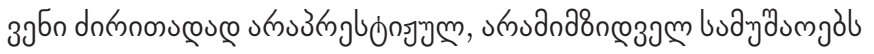

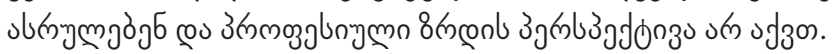

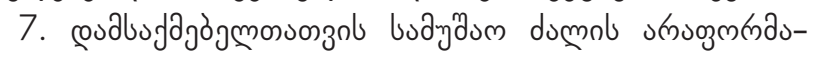

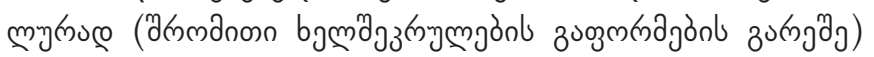

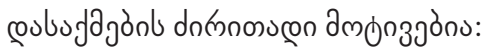

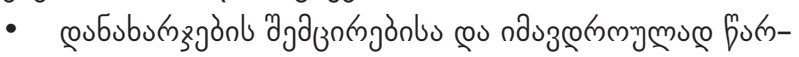

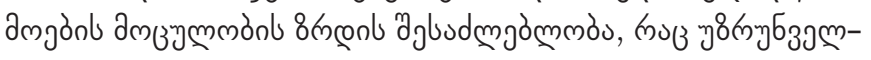

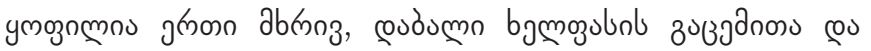

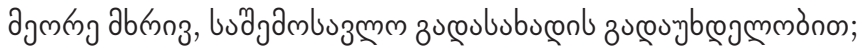

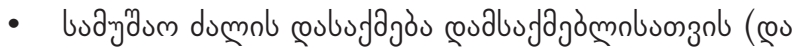

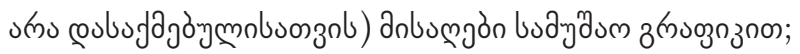

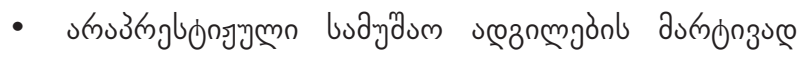

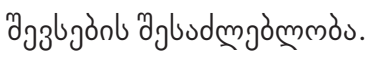

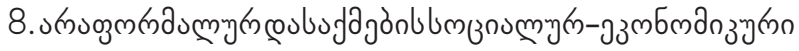

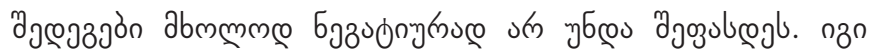

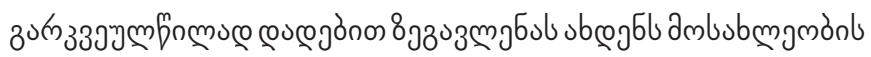

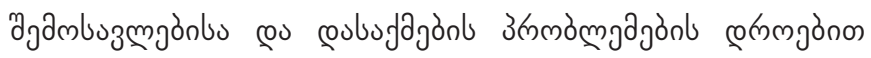
зuณ्ufy

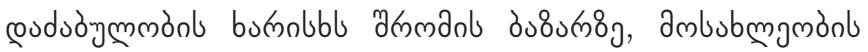

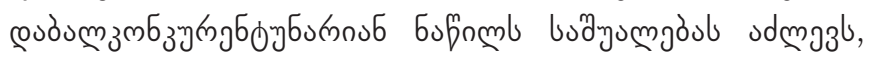

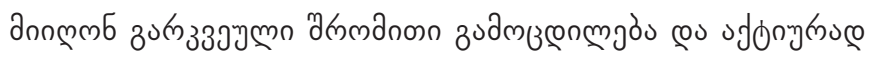

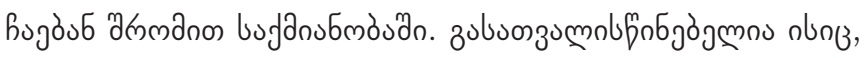

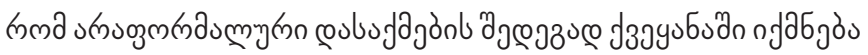

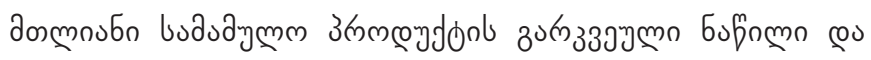

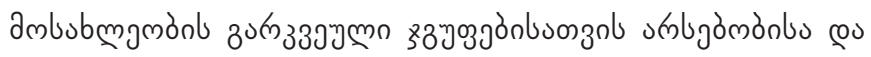
muznl zucoumh

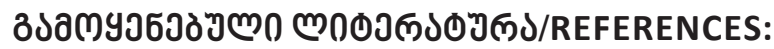

Abesadze, R. (2020). Globalization and the Economic Problems of the Pandemic. Ekonomisti, (2). ek_4_2020_bolo.pdf.

Bedianashvili, G. (2018). Culture as a factor in the formation of the knowledge economy in the systemic institutional context of paradigm. Globalization and Business, 6, 58-66. https://doi.org/10.35945/gb.2018.06.007.

Bedianashvili, G., \& Maglakelidze, A. (2021). The Digital Economy and Business in the Conditions of Pandemic. The materials of the International Scientific Conference: Social Sciences for Regional Development 2020. Part III. Issues of Economics, $26-37$.

Dzneladze, D. (2000). Employment, Labour and Social Protection of the Population. Tbilisi.

Fedorova, E.P. (2014). Informal employment in Russia: trend and causes №1, Economics and ecological management. http:// economics.open-mechanics.com/articles/1050.pdf;

Gaganidze, G. (2018). Expert Aspects of Country Competitiveness Assessment. Globalization and Business. 6, 36-42. https:// doi.org/10.35945/gb.2018.06.003;

Gelashvili, S., Muchiashvili, M., \& Maisuradze, I. (2012). Macroeconomic statistic, Tbilisi. makroekonomikuri statistik.pdf;

Gvelesiani, R. (2010). Labour market and employment policy contradictions and success strategies, Economic and Business, 4(3).

Gvelesiani, R. (2021). Problems of the Optimal Use of Economic Policy Instruments. Ecoforum, 10(2). http://www.ecoforumjournal.ro/index.php/eco/article/view/ 1250/756;

ILO (05 May 2020). COVID-19 crisis and the informal economy: Immediate responses and policy challenges. https://www.ilo.org/;

ILO (2018). Women and Men in the Informal Economy: A Statistical Picture Third Edition https://www.ilo.org/global/publications/books/WCMS_626831/lang--en/index.htm;

ILO (2020). KILM 8. Employment in the informal economy. ilo.org;

ILO Standards and Covid-19 (coronavirus), (2020). FAQ: Key provisions of international labour standards relevant to the evolving COVID-19 outbreak, Department of Norms of the Office (NORMES);

Informal employment in Ukraine: dimension, scale, dynamics. (2020). www.myshared.ru/slide/539512;

International Labour Organization, (2018). https://www.ilo.org/moscow/news/WCMS_6277 98/lang-ru/index.htm;

Kharaishvili, E., \& Natsvlishvili, I. (2019). Impact of International Educational Migration in the Context of Globalization of Higher Education (Case of Georgia). http://dspace.tsu.ge/xmlui/handle/123456789/299;

Labor in the world (1996): (ILO). Geneva, Moscow. 8.

Latsabidze, N. (1999). Socio-Economic aspects of informal employment. Social Economic.

Law of Georgia. (2020, 14 July). On employment promotion, Tbilisi, 6819.

Mekvabishvili, E. (2019). The Economic Role of the State in the Context of Globalization. Globalization and Business, 8, 22-33. https://doi.org/10.35945/gb.2019.08.002;

Papava, V. (2015). Economic of Georgia. Reforms and pseudo-reforms. Publisher, Intelekti, Tbilisi.

Papava, V. (2020, March 21). Coronomic and Economics in the Face of Crisis. https://for.ge/view/ 182671/koronomika-da-ekonomika-krizisis-winaSe.html; 
Papava, V. (2019, May 3). If there is Economic Growth, Where is the Population Fleeing from?! (In Georgian). http://bfm.ge/ lado-papava-tu-ekonomikuri-zrdaa-sad-garbis-mosakhleoba/

Papava, V., \& Charaia, V. (2020). The Coronomic Crisis and Some Challenges of the Georgian Economy. Expert opinion. 136. Georgian Strategy and International Relations Research Foundation.

Research: Informal Employment during the Pandemic Decreased by 925 Thousand People. (2020). banki.ru;

Resolution Government of Georgia. (2016, 4 April). On approval active labour market policy strategy and the action plan for its implementation in 2016-2018, 167.

Resolution Government of Georgia. (2013, 2 august). On approval of the state strategy for the formation of Georgian labour market and action plan 2015-2018 for the implementation of the state strategy for the formation of the Georgian labour market, 199, Tbilisi.

Resolution Government j;l;jumny7jk yu;ljkk yuk j l;k;ljky7uukk yuuum of Georgia. (2021, 8 January). On the approval of the 2021 state program for the development of employment promotion services Tbilisi, 2.

Resolution Government of Georgia. (2014, 17 June). On approval and some related measures Georgian Socio-Economic development strategy - Georgia 2020, Tbilisi, 400.

Resolution Government of Georgia. (2019, 30 December), on approval of the national strategy of labour and employment policy of Georgia for 2019-2023, 662.

Shelia, M. (2014). Employers Demand on Labour Force in Georgia's Big Cities. Interdisciplinary Perspectives on Social Sciences. Cambridge Scholars Publishing, UK, 113-118.

Silagadze, A., \& Gelashvili, S. (2007). Die entwicklung der finanzpolitik in Georgien. FinanzwissenschaftlicheDiskussionsbeiträge, G-2, 1-8. http://eprints.tsu.ge/1101/.

Silagadze, A., Atanelishvili, T., \& Silagadze, N. (2020). Some Tendencies of Post-Soviet Migration and Unemployment. Bulletin of the Georgian National Academy of Sciences, 14(1),172-176. http://science.org.ge/bnas/t14-n1/27_Silagadze_Economis.pdf;

Tsartsidze, M. (2019). Unemployment and the Effective Employment Problems in Georgia under the Modern Globalization Terms. Institute of Researches and International. Journal of International Economic Research. 4 (1). http://irissymposium. wixsite.com/website;

Tsartsidze, M., \& Latsabidze, N. (2020). Structural and Sectoral Features of the Labour Market and Employment in Georgia. Social and Labor Research, 38(1):153-163. https://doi.org/10.34022/2658-3712-2020-38-1-153-163. (cyberleninka.ru);

Tsartsidze, M., (2004). Labor market formation and reproduction of the workforce in Georgia. Doctoral dissertation. TSU, Tbilisi.

Tsartsidze, M., (2019). Effective Employment-The Key Factor in Human Welfare and Economic Growth in Georgia. Ekonomisti, 2. http://ekonomisti.tsu.ge/?cat=nomer\&leng=ge.

Tukhashvili, M. (2012). Georgian Labour Market in the Condition of Political and Economic Crisis. Youth Employment: Challenges and Opportunities. Timisoara.Eurostampa, 294-300.

Tukhashvili, M., Ananiashvili, I., Tsartsidze, M., Latsabidze, N., Shelia, M., \& Chelidze, N. (2012). Labour Market and Reintegration of Returned Migrants in Georgia. The publication is prepared by Danish refugee council project - Reintegration Activities in Georgia. Publisher Universal, Tbilisi.

Tukhashvili., M., \& Shelia, M. (2012). The Impact of Labour Emigration on the Demographic and Economic Development of Georgia in the Post-Soviet Period. http://www.carim-east.eu/media/CARIM-East-2012-RR-29.pdf; 